\title{
THE GLOBAL DYNAMICS OF A QUARTIC DIFFERENCE EQUATION
}

\author{
JASMIN BEKTEŠEVIĆ ${ }^{1}$, FATIH DESTOVIĆ ${ }^{2 *}$, VAHIDIN HADŽIABDIĆ ${ }^{1}$, MIDHAT \\ MEHULJIĆ ${ }^{1}$ \\ ${ }^{1}$ University of Sarajevo, Faculty of Mechanical Engineering, Division of Mathematics, Sarajevo, \\ Bosnia and Herzegovina \\ ${ }^{2}$ University of Sarajevo, Faculty of Educational Sciences, Sarajevo, Bosnia and Herzegovina \\ *Corresponding author, e-mail: fatih_d@msn.com
}

DOI: $10.20948 /$ mathmontis-2020-47-4

Summary. In this paper we will present the global dynamic and the Julia set of a quartic second order difference equation with nonnegative parameters and the initial conditions are arbitrary nonnegative real numbers.

\section{INTRODUCTION}

In general, polynomial difference equations and polynomial maps in the plane have been studied in both the real and complex domains (see [8,9]). First results on quadratic polynomial difference equation have been obtained in $[1,2]$ but these results gave us only a part of the basins of attraction of equilibrium points and period-two solutions. In [4], the general second order difference equation is completely investigated and described the regions of initial conditions in the first quadrant for which all solutions tend to equilibrium points, period-two solutions, or the point at infinity, except for the case of infinitely many period-two solutions. In [3], case of infinitely many period-two solutions is completely investigated. Our results are based on the theorems which hold for monotone difference equations. Our principal tool is the theory of monotone maps, and in particular cooperative maps, which guarantee the existence and uniqueness of the stable and unstable invariant manifolds for the fixed points and periodic points (see [5]). Consider the difference equation

$$
x_{n+1}=f\left(x_{n}, x_{n-1}\right) ; n=0,1, \ldots
$$

where $f$ is a continuous and increasing function in both variables. The following result has been obtained in [1]:

Theorem 1 Let $I \subseteq R$ and let $f \in C[I \times I, I]$ be a function which increases in both variables. Then for every solution of Eq. (1) the sub sequences $\left\{x_{2 n}\right\}_{n=0}^{\infty}$ and $\left\{x_{2 n+1}\right\}_{n=-1}^{\infty}$ of even and odd terms of the solution do exactly one of the following:

(i) Eventually they are both monotonically increasing.

(ii) Eventually they are both monotonically decreasing.

(iii) One of them is monotonically increasing and the other is monotonically decreasing

2010 Mathematics Subject Classification: 39A05, 39A10, 39A23

Keywords and Phrases: Basin of Attraction, Period-two solutions, Julia set, Difference equation, stable and unstable manifold, invariant manifolds 
As a consequence of Theorem 1 every bounded solution of Eq. (1) approaches either an equilibrium solution or period-two solution and every unbounded solution is asymptotic to the point at infinity in a monotonic way. Thus, the major problem in dynamics of Eq. (1) is the problem of determining the basins of attraction of three different types of attractors: the equilibrium solutions, period-two solution(s) and the point(s) at infinity. The following result can be proved by using the techniques of proof of Theorem 11 in [5].

Theorem 2 Consider Eq. (1) where $\mathrm{f}$ is increasing function in its arguments and assume that there is no minimal period-two solution. Assume that $E_{1}\left(x_{1}, y_{1}\right)$ and $E_{2}\left(x_{2}, y_{2}\right)$ are two consecutive equilibrium points in North-East ordering that satisfy

$$
\left(x_{1}, y_{1}\right) \preccurlyeq_{n e}\left(x_{2}, y_{2}\right)
$$

and that $E_{1}$ is a local attractor and $E_{2}$ is a saddle point or a non-hyperbolic point with second characteristic root in interval $(-1,1)$, with the neighborhoods where $\mathrm{f}$ is strictly increasing. Then the basin of attraction $B\left(E_{1}\right)$ of $E_{1}$ is the region below the global stable manifold $W^{S}\left(E_{2}\right)$. More precisely

$$
B\left(E_{1}\right)=\left\{(x, y): \exists y_{u}: y<y_{u},\left(x, y_{u}\right) \in W^{s}\left(E_{2}\right)\right\} .
$$

The basin of attraction $B\left(E_{1}\right)=W^{s}\left(E_{2}\right)$ is exactly the global stable manifold of $E_{2}$. The global stable manifold extends to the boundary of the domain of Eq. (1). If there exists a period-two solution, then the end points of the global stable manifold are exactly the period two solution.

Now, the theorems that are applied in [5] provided the two continuous curves $W^{s}\left(E_{2}\right)$ (sta-ble manifold) i $W^{u}\left(E_{2}\right)$ (unstable manifold), both passing through the point $E_{2}\left(x_{2}, y_{2}\right)$ from Theorem 2, such that $W^{s}\left(E_{2}\right)$ is a graph of decreasing function and $W^{u}\left(E_{2}\right)$ is a graph of an increasing function. The curve $W^{s}\left(E_{2}\right)$ splits the first quadrant of initial conditions into two disjoint regions, but we do not know the explicit form of the curve $W^{u}\left(E_{2}\right)$. In this paper we investigate the following difference equation

$$
\begin{gathered}
x_{n+1}=a x_{n}^{3} x_{n-1}+b x_{n}^{2} x_{n-1}^{2}+b x_{n} x_{n-1}^{3}+a x_{n-1}^{4}+c x_{n}^{2} x_{n-1}+d x_{n} x_{n-1}^{2}+ \\
+c x_{n-1}^{3}+e x_{n} x_{n-1}+e x_{n-1}^{2}+f x_{n-1}, n=0,1, \ldots
\end{gathered}
$$

We expose the explicit form of the curve that separates the first quadrant into two basins of attraction of a locally stable equilibrium point and of the point at infinity. One of the major problems in the dynamics of polynomial maps is determining the basin of attraction of the point at infinity and in particular the boundary of the that basin known as the Julia set. We precisely determined the Julia set of Eq. (2) and we obtained the global dynamics in the interior of the Julia set, which includes all the points for which solutions are not asymptotic to the point at infinity. It turned out that the Julia set for Eq. (2) is the union of the stable manifolds of some saddle equilibrium points, nonhyperbolic equilibrium points or period-two points. We first list some results needed for the proofs of our theorems. The main result for studying local stability of equilibrium is linearized stability theorem (see Theorem 1.1 in [7]).

Theorem 3 (linearized stability): Consider the difference equation

$$
x_{n+1}=f\left(x_{n}, x_{n-1}\right) ; n=0,1, \ldots
$$


and let $\bar{x}$ be an equilibrium point of difference equation (3). Let $p=\frac{\partial f(\bar{x}, \bar{x})}{\partial u}$ and $q=\frac{\partial f(\bar{x}, \bar{x})}{\partial v}$ denote the partial derivatives of $f(u, v)$ evaluated at equilibrium $\bar{x}$. Let $\lambda_{1}$ and $\lambda_{2}$ be roots of quadratic equation $\lambda^{2}-p \lambda-q=0$.

a) If $\left|\lambda_{1}\right|<1$ and $\left|\lambda_{2}\right|<1$, then the equilibrium $\bar{x}$ is locally asymptotically stable (sink).

b) If $\left|\lambda_{1}\right|>1$ or $\left|\lambda_{2}\right|<1$, then the equilibrium $\bar{x}$ is unstable.

c) $\left|\lambda_{1}\right|<1$ and $\left|\lambda_{2}\right|<1 \Leftrightarrow|p|<1-q<2$. Equilibrium $\bar{x}$ is a sink.

d) $\left|\lambda_{1}\right|>1$ and $\left|\lambda_{2}\right|>1 \Leftrightarrow|q|>1$ and $|p|<|1-q|$. Equilibrium $\bar{x}$ is a repeller.

e) $\left|\lambda_{1}\right|>1$ and $\left|\lambda_{2}\right|<1 \Leftrightarrow|p|>|1-q|$. Equilibrium $\bar{x}$ is a saddle point.

f) $\left|\lambda_{1}\right|=1$ or $\left|\lambda_{2}\right|=1 \Leftrightarrow|p|=|1-q|$ or $q=-1$ and $|p| \leq 2$. Equilibrium $\bar{x}$ is called a non-hyperbolic point.

The next theorem (Theorem 1.4.1. in [6]) is a very useful tool in establishing bounds for the solutions of nonlinear equations in terms of the solutions of equations with known behaviour.

Theorem 4 Let $\mathrm{I}$ be an interval of real numbers, let $\mathrm{k}$ be a positive integer, and let $F: I^{k+1} \rightarrow I$ I be a function which is increasing in all its arguments. Assume that $\left\{x_{n}\right\}_{n=-\mathrm{k}}^{\infty}$, $\left\{y_{n}\right\}_{n=-\mathrm{k}}^{\infty}$ and $\left\{z_{n}\right\}_{n=-\mathrm{k}}^{\infty}$ are sequences of real numbers such that

$$
\begin{aligned}
& x_{n+1} \leq F\left(x_{n}, \ldots, x_{n-k}\right), n=0,1, \ldots \\
& y_{n+1}=F\left(y_{n}, \ldots, y_{n-k}\right), n=0,1, \ldots \\
& z_{n+1} \geq F\left(z_{n}, \ldots, z_{n-k}\right), n=0,1, \ldots
\end{aligned}
$$

and

$$
x_{n} \leq y_{n} \leq z_{n}, \quad \text { for all }-k \leq n \leq 0
$$

Then

$$
x_{n} \leq y_{n} \leq z_{n}, \quad \text { for all } n>0
$$

The next well-known theorem gives us the number of positive zeros of a polynomial $P(x)$.

Theorem 5 Let $P(x)=a_{0} x^{b_{0}}+a_{1} x^{b_{1}}+\cdots+a_{n} x^{b_{n}}$ where $a_{i}, i=0,1, \ldots, n$ are real numbers and $0 \leq b_{0}<b_{1}<\cdots<b_{n}$ are integers. The number of positive zeros of $P(x)=0$, counting multiplicities, is either equal to $v(P)$ or less than that by an even number, where $v(P)$ denotes the number of sign changes in the sequence $a_{0}, a_{1}, \ldots, a_{n}$. 


\section{MAIN RESULTS}

By using the Theorem 3, we obtained the following result on local stability of the zero equilibrium of Eq. (2):

Proposition 1 The zero equilibrium of Eq. (2) is one of the following:
a) locally asymptotically stable if $\mathrm{f}<1$,
b) non-hyperbolic and locally stable if $\mathrm{f}=1$,
c) unstable if $\mathrm{f}>1$.

Set $f(x, y)=a x^{3} y+b x^{2} y^{2}+b x y^{3}+a y^{4}+c x^{2} y+d x y^{2}+c y^{3}+e x y+e y^{2}+f y$ and let $p=\frac{\partial f(\bar{x}, \bar{x})}{\partial u}$ and $q=\frac{\partial f(\bar{x}, \bar{x})}{\partial v}$ denote the partial derivatives of $f(x, y)$ evaluated at the equilibrium $\bar{x}$. The linearized equation at the positive equilibrium $\bar{x}$ is

$$
\begin{gathered}
z_{n+1}=p z_{n}+q z_{n-1} \\
p=3(a+b) \bar{x}^{3}+(2 c+d) \bar{x}^{2}+e \bar{x} \\
p=5(a+b) \bar{x}^{3}+(4 c+2 d) \bar{x}^{2}+3 e \bar{x}+f .
\end{gathered}
$$

Now, in view of Theorem 3 we obtain the following results on local stability of the positive equilibrium of Eq. (2):

Proposition 2 The positive equilibrium of Eq. (2) is one of the following:
a) locally asymptotically stable if $p+q<1$,
b) non-hyperbolic and locally stable if $p+q=1$,
c) unstable if $p+q>1$,
d) saddle point if $p>|q-1|$,
e) repeller if $1-\mathrm{q}<p<q-1$.

Theorem 6 If $f \geq 1$ then every solution $\left\{x_{n}\right\}$ of Eq. (2) satisfieslim $\lim _{n \rightarrow \infty} x_{n}=\infty$.

Proof. If $\left\{x_{n}\right\}$ is a solution of Eq. (2) then $\left\{x_{n}\right\}$ satisfies the inequality

$$
x_{n+1} \geq f x_{n-1}, n=0,1, \ldots
$$

which in view of the result on difference inequalities, see Theorem 4 , implies that $x_{n} \geq$ $y_{n}, n \geq 1$ where $\left\{x_{n}\right\}$ is a solution of the initial value problem

$$
y_{n+1} f y_{n-1}, y_{-1}=x_{-1} \text { and } y_{0}=x_{0} n=0,1, \ldots
$$


Consequently, $x_{0}, x_{-1}>0$ then $y_{0}, y_{-1}>0, y_{n} \geq 0$ for all $n$, and

$$
x_{\mathrm{n}} \geq y_{n}=\lambda_{1} \sqrt{f}^{n}+\lambda_{2}(-\sqrt{f})^{n}, n=1,2, \ldots
$$

where $\lambda_{1}, \lambda_{2} \in \boldsymbol{R}$ such that $y_{n} \geq 0$ for all $n$, which implies $\lim _{n \rightarrow \infty} x_{n}=\infty$.

Theorem 7 Consider the difference equation (2) in the first quadrant of initial conditions, where a $>0, b, c, d, e, f \geq 0$ and $2 \mathrm{e}+\mathrm{f}<1$. Then Eq. (2) has a zero equilibrium and a unique positive equilibrium $\bar{x}_{+}$. The line $a x^{3}+b x^{2} y+b x y^{2}+a y^{3}+c x^{2}+d x y+$ $c y^{2}+e x+e y+f=1$ is the Julia set and separates the first quadrant into two regions: the region below the line is the basin of attraction of point $E_{0}(0,0)$ the region above the line is the basin of attraction of the point at infinity and every point on the line except $E_{+}\left(x_{+}, x_{+}\right)$ is a period-two solution of Eq.(2)

Proof. The equilibrium points of Eq. (2) are the solutions of equation

$$
2(a+b) x^{4}+(2 c+d) x^{3}+2 e x^{2}+f x=x
$$

that is equivalent to

$$
\left(2(a+b) x^{3}+(2 c+d) x^{2}+2 e x+f-1\right) x=0
$$

Since the number of sign changes in the sequence $2(a+b), 2 c+d, 2 e, f-1$ is one, then by applying Theorem 5 implies Eq. (4) has two equilibria: zero equilibrium and unique positive equilibrium $\mathrm{x}_{+}$. Since $f \geq 0$ and $\mathrm{f}<1$, then by applying Proposition (1) the zero equilibrium is locally asymptotically stable. Denote by $h(x ;, y)=a x^{3} y+b x^{2} y^{2}+$ $b x y^{3}+a y^{4}+c x^{2} y+d x y^{2}+c y^{3}+e x y+e y^{2}+f y$ and let $p$ and $q$ denote the partial derivatives of function $h(x, y)$ at point $E_{+}$. By straightforward calculation we obtain that the following hold:

$$
\begin{gathered}
p+q=\left(3(a+b) \bar{x}^{3}+(2 c+d) \bar{x}^{2}+e \bar{x}\right)+\left(5(a+b) \bar{x}^{3}+(4 c+2 d) \bar{x}^{2}+3 e \bar{x}+f\right) \\
=8(a+b) \bar{x}^{3}+(6 c+3 d) \bar{x}^{2}+4 e \bar{x}+f \\
=2 \bar{x}\left(3(a+b) \bar{x}^{2}+(2 c+d) \bar{x}+e\right)+\left(2(a+b) \bar{x}^{3}+(2 c+d) \bar{x}^{2}+2 e \bar{x}+f\right) \\
=2 \bar{x}\left(3(a+b) \bar{x}^{2}+(2 c+d) \bar{x}+e\right)+1>1 . \\
q-p=\left(5(a+b) \bar{x}^{3}+(4 c+2 d) \bar{x}^{2}+3 e \bar{x}+f\right)-\left(3(a+b) \bar{x}^{3}+(2 c+d) \bar{x}^{2}+e \bar{x}\right) \\
=\left(2(a+b) \bar{x}^{3}+(2 c+d) \bar{x}^{2}+2 e \bar{x}+f-1\right)+1=1 .
\end{gathered}
$$

Hence, by applying Proposition (2) the positive equilibrium is an unstable non-hyperbolic point. Period-two solution $u, v$ satisfies the system

$$
\begin{aligned}
& u=\left(a v^{3}+b u v^{2}+b u^{2} v+a u^{3}+c v^{2}+d u v+c u^{2}+e v+e u+f\right) u \\
& v=\left(a u^{3}+b u^{2} v+b u v^{2}+a v^{3}+c u^{2}+d u v+c v^{2}+e u+e v+f\right) v .
\end{aligned}
$$

Obviously, the point $(0,0)$ is solution of the system above, but it is not period two solution. Hence, it has to be $v>0$ which implies

$$
a u^{3}+b u^{2} v+b u v^{2}+a v^{3}+c u^{2}+d u v+c v^{2}+e u+e v+f=1 .
$$


Therefore every point of the set

$$
\left\{(x, y): a x^{3}+b x^{2} y+b x y^{2}+a y^{3}+c x^{2}+d x y+c y^{2}+e x+e y+f=1\right\}
$$

is a period-two solution of Eq. (2) except point $E_{+}$. Now, we have to show that line

$$
\begin{gathered}
g(x, y)=a x^{3}+b x^{2} y+b x y^{2}+a y^{3}+c x^{2}+d x y+c y^{2}+e x+e y \\
+f=1
\end{gathered}
$$

is a graph of the decreasing function in the first quadrant. Let for some $\mathrm{x}>0$ there are $\mathrm{y}_{1}$ and $\mathrm{y}_{2}\left(0<\mathrm{y}_{1}<\mathrm{y}_{2}\right)$ such that $g\left(x, y_{1}\right)=g\left(x, y_{2}\right)=1$. As $g(x, y)$ is increasing in both variables then

$$
1=g\left(x, y_{1}\right)<g\left(x, y_{2}\right)=1,
$$

which is impossible. Thus the curve $g(x, y)=1$ is the graph of function in the first quadrant. Further over $g(x, y)=1$ then

$$
\frac{\partial g}{\partial x}+\frac{\partial g}{\partial y} y^{\prime}=0
$$

By applying the fact that is $g(x, y)$ is increasing in both variables we obtain $y^{\prime}<0$ in the first quadrant. Hence, $g(x, y)=1$ is the graph of the decreasing function in the first quadrant. Let $\left\{x_{n}\right\}$ be a solution of Eq. (2) for initial condition $\left(x_{0}, x_{-1}\right)$ which lies below the line

$$
a x^{3}+b x^{2} y+b x y^{2}+a y^{3}+c x^{2}+d x y+c y^{2}+e x+e y+f=1 .
$$

Then

$$
\begin{gathered}
g\left(x_{0}, x_{-1}\right)=a x_{0}^{3}+b x_{0}^{2} x_{-1}+b x_{0} x_{-1}^{2}+a x_{-1}^{3}+c x_{0}^{2}+d x_{0} x_{-1}+c x_{-1}^{2}+e x_{0} \\
+e x_{-1}+f<1, \\
x_{n+1}=g\left(x_{n}, x_{n-1}\right) x_{n-1}
\end{gathered}
$$

and

$$
\begin{gathered}
x_{1}=g\left(x_{0}, x_{-1}\right) x_{-1}<x_{-1}, \\
x_{2}=g\left(x_{1}, x_{0}\right) x_{0}<g\left(x_{-1}, x_{0}\right) x_{0}=g\left(x_{0}, x_{-1}\right) x_{0}<x_{0} .
\end{gathered}
$$

Thus $\left(x_{2}, x_{1}\right)$ and $\left(x_{0}, x_{-1}\right)$ are two points in North-East ordering $\left(x_{2}, x_{1}\right) \leq_{n e}\left(x_{0}, x_{-1}\right)$ which means that the point $\left(x_{2}, x_{1}\right)$ is also below the line $g(x, y)=1$ and also holds

$$
g\left(x_{2}, x_{1}\right)<1
$$

Similarly, we find

$$
\begin{gathered}
x_{3}=g\left(x_{2}, x_{1}\right) x_{1}<x_{-1}, \\
x_{4}=g\left(x_{3}, x_{2}\right) x_{2}<g\left(x_{1}, x_{2}\right) x_{2}=g\left(x_{2}, x_{1}\right) x_{2}<x_{2} .
\end{gathered}
$$

Continuing on this way we get

$$
(0,0) \leq_{n e} \ldots \leq_{n e}\left(x_{4}, x_{3}\right) \leq_{n e}\left(x_{2}, x_{1}\right) \leq_{n e}\left(x_{0}, x_{-1}\right)
$$

which implies that both sub sequences $\left\{x_{2 n}\right\}$ and $\left\{x_{2 n+1}\right\}$ are monotonically decreasing and bounded below by 0 . Since below the line $g(x, y)=1$ there are no period-two solutions it must be $x_{2 n} \rightarrow 0$ and $x_{2 n+1} \rightarrow 0$. On the other hand, if we consider solution $\left\{x_{2 n}\right\}$ of Eq.(2) 
for initial condition $\left(x_{0}, x_{-1}\right)$ which lies above the line $g(x, y)=1$ then $g\left(x_{0}, x_{-1}\right)>1$ and by applying the method shown above we obtain the following condition:

$$
\left(x_{-1}, x_{0}\right) \leq_{n e}\left(x_{1}, x_{2}\right) \leq_{n e}\left(x_{3}, x_{4}\right) \leq_{n e} \ldots
$$

Therefore, both sub sequences $\left\{x_{2 n}\right\}$ and $\left\{x_{2 n+1}\right\}$ are monotonically increasing, hence $x_{2 n} \rightarrow \infty$ and $x_{2 n+1} \rightarrow \infty$ as $\mathrm{n} \rightarrow \infty$.

The next figure is visual illustration of Theorem 7 obtained by using Mathematica 9.0, with the boundaries of the basins of attraction obtained by using the software package Dynamica [6].

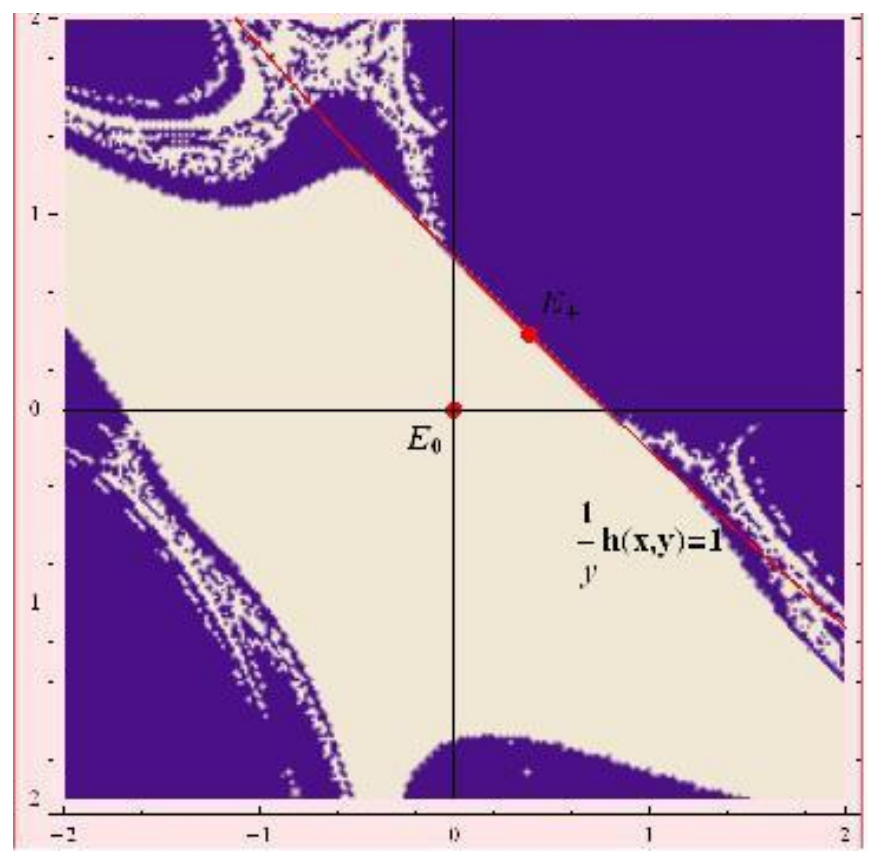

Figure 1. Illustration of Theorem 7

$$
\mathrm{a}=0.3, \mathrm{~b}=1, \mathrm{c}=0.5, \mathrm{~d}=1, \mathrm{e}=0.4 \text { and } \mathrm{f}=0.25
$$

In view of Theorem 4 which implies results on difference inequalities we get the following:

Proposition 3 Consider the difference equation of type

$$
\begin{gathered}
x_{n+1}=A x_{n}^{3} x_{n-1}+B x_{n}^{2} x_{n-1}^{2}+C x_{n} x_{n-1}^{3}+D x_{n-1}^{4}+E x_{n}^{2} x_{n-1}+F x_{n} x_{n-1}^{2}+ \\
+G x_{n-1}^{3}+H x_{n} x_{n-1}+I x_{n-1}^{2}+J x_{n-1},
\end{gathered}
$$

where the given parameters satisfy conditions $A, B, C, D, E, F, G, H, I>0$ and $0<J<1$. Then the global stable manifold of the positive equilibrium is between two lines

$$
\begin{aligned}
p_{1}: \min \{A, D\}\left(x^{3}+y^{3}\right) & +\min \{B, C\}\left(x^{2} y+x y^{2}\right)+\min \{E, G\}\left(x^{2}+y^{2}\right) \\
& +\min \{H, I\}(x+y)+F x y+J=1 .
\end{aligned}
$$

and 


$$
\begin{gathered}
p_{2}: \max \{A, D\}\left(x^{3}+y^{3}\right)+\max \{B, C\}\left(x^{2} y+x y^{2}\right)+\max \{E, G\}\left(x^{2}+y^{2}\right) \\
+\max \{H, I\}(x+y)+F x y+J=1 .
\end{gathered}
$$

Proof. Since the number of sign changes in the sequence $A+B+C+D, E+F+$ $G, H+I, J-1$ is one, then by applying Theorem 5 implies Eq. (5) has two equilibria: zero equilibrium and unique positive equilibrium $\bar{x}_{+}$. Since $J<1$ the zero equilibrium is always locally asymptotically stable thus the positive equilibrium must be unstable equilibrium point. The theorems applied in [5] provided the following global behavior. More precisely, if the positive equilibrium is a saddle point or a non-hyperbolic point then there exists a global stable manifold which contains point $E_{+}(\bar{x}, \bar{x})$; where $\bar{x}$ is the positive equilibrium. In this case global behavior of Eq. (5) is described by Theorem 9 in [4]. If the positive equilibrium is a repeller then there exists a period-two solution and we obtain that the period-two solution is a saddle point and there are two global stable manifolds which contain points $P_{1}(u, v)$ and $P_{2}(v, u)$ whre $(u, v)$ is unique period-two solution of Eq.(5). In this case the global behavior of Eq. (5) is described by Theorem 10 in [4]. Although the Theorems 9 and 10 in [4] have been applied on a polynomial second order difference equation they are special cases of general Theorems in [5] applied on function $\mathrm{f}$, where $\mathrm{f}$ is increasing function in its arguments. So, the global dynamics of Eq. (5) is exactly the same as the global dynamics of equations described by Theorems 9 and 10 in [4]. Furthermore

$$
\begin{gathered}
x_{n+1}=A x_{n}^{3} x_{n-1}+B x_{n}^{2} x_{n-1}^{2}+C x_{n} x_{n-1}^{3}+D x_{n-1}^{4}+E x_{n}^{2} x_{n-1}+F x_{n} x_{n-1}^{2}+ \\
+G x_{n-1}^{3}+H x_{n} x_{n-1}+I x_{n-1}^{2}+J x_{n-1} \\
\geq\left(\min \{A, D\}\left(x_{n}^{3}+x_{n-1}^{3}\right)+\min \{B, C\}\left(x_{n}^{2} x_{n-1}+x_{n} x_{n-1}^{2}\right)\right) x_{n-1}+ \\
+\left(\min \{E, G\}\left(x_{n}^{2}+x_{n-1}^{2}\right)+\min \{H, I\}\left(x_{n}+x_{n-1}\right)+F x_{n} x_{n-1}+J\right) x_{n-1},
\end{gathered}
$$

and

$$
\begin{gathered}
x_{n+1}=A x_{n}^{3} x_{n-1}+B x_{n}^{2} x_{n-1}^{2}+C x_{n} x_{n-1}^{3}+D x_{n-1}^{4}+E x_{n}^{2} x_{n-1}+F x_{n} x_{n-1}^{2}+ \\
+G x_{n-1}^{3}+H x_{n} x_{n-1}+I x_{n-1}^{2}+J x_{n-1} \\
\leq\left(\max \{A, D\}\left(x_{n}^{3}+x_{n-1}^{3}\right)+\max \left(x_{n}^{2} x_{n-1}+x_{n} x_{n-1}^{2}\right)\right) x_{n-1}+ \\
+\left(\max \{E, G\}\left(x_{n}^{2}+x_{n-1}^{2}\right)+\max \{H, I\}\left(x_{n}+x_{n-1}\right)+F x_{n} x_{n-1}+J\right) x_{n-1} .
\end{gathered}
$$

for all $n$, by applying Theorem 4 for solution $\left\{x_{n}\right\}$ of Eq. (5) the following inequality holds

$$
y_{n} \leq x_{n} \leq z_{n}
$$

for all $n$, where $\left\{y_{n}\right\}$ is a solution of the difference equation

$$
\begin{aligned}
& y_{n+1}=\left(\min \{A, D\}\left(y_{n}^{3}+y_{n-1}^{3}\right)+\min \{B, C\}\left(y_{n}^{2} y_{n-1}+y_{n} y_{n-1}^{2}\right)\right) x_{n-1} \\
& +\left(\min \{E, G\}\left(y_{n}^{2}+y_{n-1}^{2}\right)+\min \{H, I\}\left(y_{n}+y_{n-1}\right)+F y_{n} y_{n-1}+J\right) x_{n-1},
\end{aligned}
$$

and $\left\{z_{n}\right\}$ is a solution of the difference equation

$$
z_{n+1}=\left(\max \{A, D\}\left(z_{n}^{3}+z_{n-1}^{3}\right)+\max \{B, C\}\left(z_{n}^{2} z_{n-1}+z_{n} z_{n-1}^{2}\right)\right) x_{n-1}
$$




$$
+\left(\max \{E, G\}\left(z_{n}^{2}+z_{n-1}^{2}\right)+\max \{H, I\}\left(z_{n}+z_{n-1}\right)+F z_{n} z_{n-1}+J\right) x_{n-1} .
$$

Since Eq. (8) and Eq. (9) satisfy all conditions of Theorem 7 this implies that the statement of Proposition 3 holds.

\section{CONCLUSION}

In this paper we restrict our attention to certain polynomial quartic second order difference equation Eq. (2). It is important to mention that we have accurately determined the Julia set of Eq. (2) and the basins of attractions for the zero equilibrium and the positive equilibrium point. In general, all theoretical concepts which are very useful in proving the results of global attractivity of equilibrium points and period-two solutions only give us existence of global stable manifold(s) whose computation leads to very uncomfortable calculus.

Acknowledgements: The authors would like to express their sincere thanks to reviewers on their valuable and constructive suggestions that contributed to this paper in clarity and form.

\section{REFERENCES}

[1] Amleh A. M., Camouzis E., Ladas G., "On the dynamics of rational difference equation, Part I", Int. J. Difference Equ., 3, 1-35 (2008).

[2] Amleh A. M., Camouzis E., Ladas G., "On the dynamics of rational difference equation, Part II", Int. J. Difference Equ., 3, 195-225 (2008).

[3] Bektešević J., Hadžiabdić V., Mehuljić M., Mujić N., "The Global Behavior of a Quadratic Difference Equation", Filomat, 32 (18), 6203-6210 (2018).

[4] Bektešević J., Kulenović M. R. S., Pilav E., "Global Dynamics of Quadratic Second Order Difference Equation in the First Quadrant", App. Math. Comp., 227, 50-65 (2014).

[5] Brett A., Kulenović M. R. S., "Basins of attraction of equilibrium points of monotone difference equations" Sarajevo J. Math., 5(18), 211-233 (2009)

[6] Camouzis E. Ladas G., Dynamics of Third Order Rational Difference Equations with Open Problems and Conjectures, Chapman and Hall/CRC Boca Raton London (2008).

[7] Kulenović M. R. S., Ladas G., Dynamics of Second Order Rational Difference Equations with Open Problems and Conjectures, Chapman and Hall/CRC Boca Raton London (2001).

[8] Milnor J., Dynamics in One Complex Variable, Stony Brook New York (2000).

[9] Morosawa S., Nishimura Y., Taniguchi M., Ueda T., Holomorphic dynamics, Cambridge University Press UK (2000).

Received January 13, 2020 